\title{
СОВЕРШЕНСТВОВАНИЕ МЕТОДИКИ КОЛИЧЕСТВЕННОЙ ОЦЕНКИ УГРОЗ ИНФОРМАЦИОННОЙ БЕЗОПАСНОСТИ ТЕЛЕКОММУНИКАЦИОННЫХ СИСТЕМ И СЕТЕЙ
}

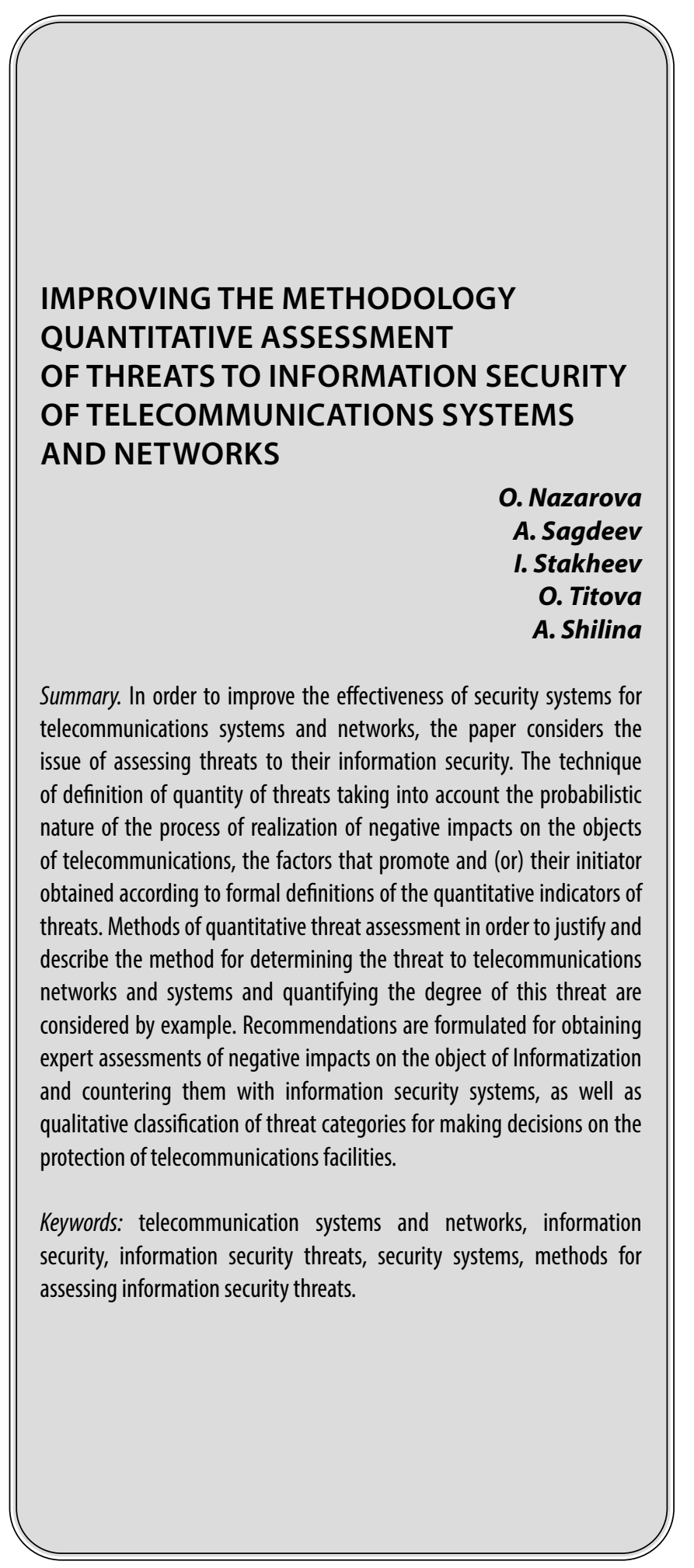

Назарова Ольга Юрьевна

К.т.н., дочент, Донской государственный технический университет, Ростов-на-Дону olga2018rostov@yandex.ru

Сагдеев Александр Константинович

К.т.н., доцент, Санкт-Петербургский государственный университет телекоммуникаций им. проф. М. А. Бонч-Бруевича brother-aks@yandex.ru

Стахеев Иван Геннадиевич

K.т.н., доцент, Санкт-Петербургский государственный университет телекоммуникаций им. проф. М.А. Бонч-Бруевича

титова Ольга Викторовна

К.т.н., дочент, Санкт-Петербургский государственный университет телекоммуникаций им. проф. М. А. Бонч-Бруевича

Шилина Анна Николаевна

К.т.н., доцент, Южно-Российский государственный политехнический университет (НПИ) имени

М.И. Платова, Новочеркасск

Аннотация. С целью повышения эффективности систем обеспечения безопасности телекоммуникационных систем и сетей, в работе рассматривается вопрос оценки угроз их информационной безопасности. Предложена методика определения количественного показателя угроз с учетом вероятностного характера процесса реализации негативных воздействий на объекты телекоммуникаций, определены факторы, способствующие и (или) их инициирующие, получены зависимости формального определения количественного показателя угроз. Методики количественной оценки угрозы с целью обоснования и описания способа определения угрозы на телекоммуникационные сети и системы и количественной оценки степени этой угрозы рассмотрены на примере. Сформулированы рекомендации по получению экспертных оценок негативных воздействий на объект информатизации и противодействия им систем обеспечения информации, а так же качественной классификации категорий угроз для принятия решений по защите объектов телекоммуникаций.

Ключевые слова: телекоммуникационные системы и сети, безопасность информации, угрозы информационной безопасности, системы обеспечения безопасности, методика оценки угроз информационной безопасности. 
B современных условиях значение телекоммуникационных систем и сетей (ТКСС), их составляющая в жизни общества, а вместе с тем важность и сложность задачи обеспечения их информационной безопасности неизмеримо возросли. Безусловно, что согласно закрепленного Федеральным законом [1] интегрального понятия защищаемого объекта, телекоммуникации становятся важной составляющей, объектом безопасности в информационной сфере.

Значимость обеспечения защиты ТКСС определяется уязвимостью, некоторым свойством информационной сферы, которое делает возможным возникновение и реализацию угрозы, негативного воздействия вследствие множества факторов, основными среди которых являются:

- огромные масштабы информационной инфраструктуры страны,

- взаимодействие объектов информатизации с международными сетями передачи и хранения информации,

возросшим числом компьютерных атак на критически важные объекты инфраструктуры,

- эксплуатация средств вычислительной техники и связи иностранного производства.

Основными объектами угрозы информационной безопасности являются объекты информатизации, информационные системы, ресурсы информационной системы, информационные технологии, программные средства и сети связи. Быстро развивающиеся технологии передачи данных требуют постоянного совершенствования методов обеспечения БИ, а значит анализа «аспектов, связанных с определением, достижением и поддержанием конфиденциальности, целостности, доступности, неотказуемости, подотчетности, аутентичности и достоверности информации или средств ее обработки» [2].

Информационной безопасностью (ИБ) ТКСС является ее способность противостоять множеству угроз, основными видами которых можно считать информационные нарушения сбора и обработки информации, аппаратно-программные угрозы и радиоэлектронные помехи, перехват информации, нормативно-правовые коллизии, а так же физические поломки, хищение и аварии в технических системах коммуникации. Применительно к основным свойствам информации возможны угрозы связанные с разглашением информации, с несанкционированным доступом, искажением (модификацией) информации, блокированием доступа к защищаемой информации, уничтожением носителей информации, непреднамеренным воздействием или сбоем оборудования и др. Виды угроз однозначно определяются видами НB на ТКСС.
Появление одного или нескольких нежелательных или неожиданных событий ИБ, имеющих значительную вероятность компрометации и указывающих на свершившуюся, преднамеренную или вероятную реализацию угрозы ИБ, является инцидентом (И) безопасности [3].

Противодействие угрозам - цель защиты систем обеспечения БИ, эффективность которых адекватно зависит от применения современных методик определения показателей угроз.

В качестве вида количественного показателя угрозы безопасности выберем степень угрозы И на объекте безопасности на некотором временном интервале $\Delta T=\left[t_{1}, t_{2}\right]$.

Учитывая вероятный характер процесса реализации И на ТКСС, степень угрозы $\beta_{j} И\left(j=1,2, \ldots, J_{i}\right)$ на $i$-й объект $(i=1,2, \ldots, I)$ количественно может характеризоваться

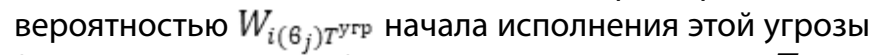
(начала реализации И) на временном интервале $\Delta \boldsymbol{T}$.

Здесь и в дальнейшем под вероятностью некоторого события, которая согласно положению теории вероятностей является неслучайной величиной, будем понимать полученную тем или иным способом ее оценку, которая представляет собой случайное число и характеризует вероятность с некоторой точностью в зависимости от способа ее определения $[4,5,6]$.

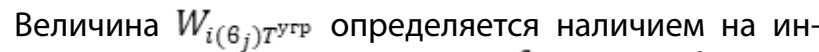
тервале $\Delta \boldsymbol{T}$ ряда факторов угрозы $f_{i j \Phi} \in \boldsymbol{F} i j, \phi=1,2, \ldots$, $\Phi_{i j}$, способствующих и/или инициирующих $\beta_{j}$ И на $i$-й объект ТКСС (1).

При этом в зависимости от своего физического содержания указанные факторы могут вызывать И как самостоятельно, так и в определенных сочетаниях.

Факторы способствующие И (и/или их инициирующие) применительно к информационной сфере:

- наличие связей объектов ТКСС с сетью Интернет;

- наличие заинтересованности определенных лиц и организаций в получении конфиденциальной информации, циркулирующей в конкретной информационной системе;

- социальная значимость ТKСС;

- уязвимостью объектов информатизации и реализуемых ими технологических процессов;

- уязвимостью систем обеспечения БИ;

- надлежащее выполнение собственниками объектов информатизации эксплуатационных требований (режим эксплуатации, техническое обслуживание, регламентные работы, ремонт и т.п.); 


$$
W_{i\left(6_{j}\right) T^{\mathrm{yrp}}}=W_{T^{\mathrm{yrp}}} f_{i j \Phi} \in F_{i j}
$$

$$
W\left(f_{i j \Phi}\right)_{\Phi}^{\mathrm{yrp}}=W\left(f_{i j \Phi}\right)^{\text {нал }}{ }_{\Phi} W\left(f_{i j \Phi}\right)^{\mathrm{B} \pi}{ }_{\Phi} .
$$

$$
\begin{aligned}
& W_{i\left(6_{j}\right) \Phi^{\mathrm{yrp}}}=\sum_{\text {Ц=1 }}^{\Phi i j} W\left(f_{i j \Phi}\right)_{T^{\mathrm{yrp}}} \sum_{{ }_{6, \mathrm{~B}}} W\left(f_{i j \sigma} \cap f_{i j_{\mathrm{B}}}\right)_{\Phi^{\mathrm{yгp}}}+\sum_{\text {бв }} W\left(f_{i j \sigma} \cap f_{i j \mathrm{~B}} \cap\right. \\
& \left.f_{i j \mathrm{r}}\right)_{\phi^{\mathrm{grp}}}-\ldots+(-1)^{\Phi_{i j-1}} W\left(f_{i j 1} \cap f_{i j 2} \cap \ldots f_{i j \Phi_{i j}}\right)_{\phi^{\mathrm{grp}}} .
\end{aligned}
$$

$$
\begin{aligned}
& W_{i}\left(\mathrm{~B}_{j}\right)_{\Phi^{\mathrm{yrp}}}=
\end{aligned}
$$

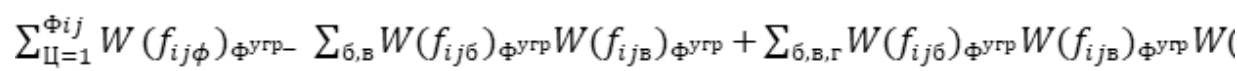

$$
\begin{aligned}
& (-1)^{\Phi_{i j-1}} W\left(f_{i j 1}\right)_{\Phi^{\mathrm{yp}}} W\left(f_{i j 2}\right)_{\Phi^{\mathrm{yrp}} \ldots W} \ldots\left(f_{i j \Phi_{i j}}\right)_{\Phi^{\mathrm{yrp}}} .
\end{aligned}
$$

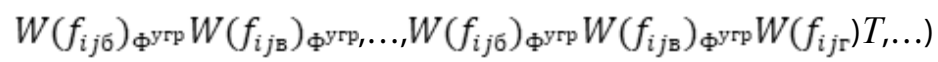

$$
W_{i\left(6_{j}\right) \Phi^{\mathrm{yrp}}}=\prod_{\phi=1}^{\Phi_{i j}} \quad\left(1-W\left(f_{i j \Phi}\right)_{\Phi^{\mathrm{yrp}}}\right)
$$

- возможность физического НД к технике и операционным системам;

- низкий уровень квалификации, состояния производственной дисциплины, морального и психологического состояния персонала объектов информатизации.

Определение перечня факторов угрозы $\boldsymbol{F}_{i j}$ их наличия на интервале $\Delta \boldsymbol{T}$ и влияния на началоИ на этом интервале является не формализуемой задачей и может осуществляться исключительно экспертными методами с участием специалистов.

В интересах формального определения степени угрозы введем в рассмотрение величину $W\left(f_{i j \Phi}\right)^{\text {нал }}$, характеризующую наличие на интервале $\Delta \boldsymbol{T}$ фактора угрозы $f_{i j \phi}$, способствующего реализации $j$-го И на $i$-й объект безопасности, и величину $W\left(f_{i j \Phi}\right)^{\text {вл }}{ }_{\Phi^{\prime}}$ характеризующую влияние этого фактора (при его наң̆ичии) на начало указанного воздействия. При введении шкалы $[0,1]$ в качестве области значений величин они могут быть интерпретированы как соответствующие вероятности.

Степень угрозы $W\left(f_{i j \Phi}\right)_{\text {угр }}$ (со стороны фактора $\left.f_{i j \Phi}\right)$ начала реализации $j$-го $V^{\Phi}$ на TKCС на интервале $\Delta \boldsymbol{T}$ определяется совпадением двух событий: наличием фактора $f_{i j \phi}$ на этом интервале и его влиянием на начало воздействия (2).
Второй сомножитель $W\left(f_{i j \Phi}\right)^{\text {вл }}$ при этом имеет смысл условной вероятности (при условия наличия фактора $f_{i j \Phi}$ на интервале $\left.\Delta \boldsymbol{T}\right)$.

В случае совместного присутствия на интервале $\Delta \boldsymbol{T}$ всей (в общем случае взаимосвязанной) совокупности факторов $f_{i j \Phi} \in \boldsymbol{F}_{i j}(\phi=1,2, \ldots, \Phi i j)$ степень угрозы начала реализации вј И на $i$-й объект ТКСС определяется следующим образом (3), где $W\left(f_{i j 6} \cap f_{i j \mathrm{~s}}\right)_{\Phi_{\text {угр }}}-$ степень угрозы, обусловленная совместным влиянием на начало вј НВ взаимосвязанных факторов $f_{i j \alpha} u f_{i j \beta}$; суммы распространяются на различные значения индексов $\phi ; \alpha, \beta ; \alpha, \beta, \gamma$ и т.д.

Если события, характеризующие присутствие на интервале $\Delta \boldsymbol{T}$ факторов $\boldsymbol{f}_{i j \phi}$ и их влияние на начало $\boldsymbol{B}_{j}$ И, являются независимыми, выражение (3) преобразуется в вид (4).

В случае, когда факторы fijp не являются независимыми, ряд соответствующих сомножителей в слагаемых зависимости (4), представляющих собой произведение вероятностей (5), будут иметь смысл условных вероятностей [7].

При использовании понятия противоположного события [8] (1-W(fijp)Тугр) зависимость (4) представляется в более компактной форме (6). 
Таблица 1

\begin{tabular}{|c|c|c|c|c|c|c|}
\hline \multirow{3}{*}{$\begin{array}{l}\text { Фактор } \\
f_{i j \phi}\end{array}$} & \multicolumn{6}{|c|}{ Инцидент на $j=1,2, \ldots, J$} \\
\hline & \multicolumn{2}{|l|}{1} & \multicolumn{2}{|l|}{$\mathrm{j}$} & \multicolumn{2}{|l|}{$\mathrm{J}$} \\
\hline & Наличие & Влияние & Наличие & Влияние & Наличие & Влияние \\
\hline 1 & $W_{k}\left(f_{i 11}\right)^{\text {нал }}$ & $W_{k}\left(f_{i 11}\right)_{\Phi}^{\mathrm{B} \pi}$ & $W_{k}\left(f_{i j 1}\right)_{\Phi}^{\text {нал }}$ & $W_{k}\left(f_{i j 1}\right)_{\Phi}^{\mathbf{B} \pi}$ & $W_{k}\left(f_{i j 1}\right)_{\Phi}^{\text {нал }}$ & $W_{k}\left(f_{i j 1}\right)_{\Phi}^{\mathbf{B} \pi}$ \\
\hline$\phi$ & $W_{k}\left(f_{i 1 \phi}\right)_{\Phi}^{\text {нал }}$ & $W_{k}\left(f_{i 1 \Phi}\right)_{\Phi}^{\mathrm{B} \pi}$ & $W_{k}\left(f_{i j \Phi}\right)_{\Phi}^{\text {нал }}$ & $W_{k}\left(f_{i j \Phi}\right)_{\Phi}^{\mathrm{B} \pi}$ & $W_{k}\left(f_{i J \Phi}\right)_{\Phi}^{\text {нал }}$ & $W_{k}\left(f_{i j \Phi}\right)_{\Phi}^{\text {влл }}$ \\
\hline$\Phi_{i j}$ & $W_{k}\left(f_{i 1 \Phi}\right)_{\Phi}^{\text {нал }}$ & $W_{k}\left(f_{i 1 \Phi}\right)^{\mathrm{B} \pi}$ & $W_{k}\left(f_{i j \Phi}\right)_{\Phi}^{\text {нал }}$ & $W_{k}\left(f_{i j \Phi}\right)_{\Phi}^{\mathrm{B} \pi}$ & $W_{k}\left(f_{i j \Phi}\right)_{\Phi}^{\text {нал }}$ & $W_{k}\left(f_{i j \Phi}\right)_{\Phi}^{\mathrm{B} \cdot \pi}$ \\
\hline
\end{tabular}

Таблица 2

\begin{tabular}{|c|c|c|c|}
\hline \multirow{2}{*}{ Фактор $f_{i j \phi}$} & \multicolumn{3}{|c|}{ Инцидент на $j=1,2, \ldots, J$} \\
\hline & 1 & j & $\mathrm{J}$ \\
\hline 1 & $W_{k}\left(f_{i 11}\right)_{\Phi^{y T p}}$ & $W_{k}\left(f_{i j 1}\right)_{\Phi^{y T p}}$ & $W_{k}\left(f_{i j 1}\right)_{\Phi^{\mathrm{yTp}}}$ \\
\hline$\phi$ & $W_{k}\left(f_{i 1 \Phi}\right)_{\Phi}$ & $W_{k}\left(f_{i j \Phi}\right)_{\Phi^{\text {yrp }}}$ & $W_{k}\left(f_{i j \Phi}\right)_{\Phi}^{y \Gamma p}$ \\
\hline$\Phi_{i j}$ & $W_{k}\left(f_{i 1 \Phi}\right)_{\Phi}$ & $W_{k}\left(f_{i j \Phi}\right)_{\Phi^{y \Gamma p}}$ & $W_{k}\left(f_{i j \Phi}\right)_{\Phi^{y \Gamma P}}$ \\
\hline
\end{tabular}

Таблица 3

\begin{tabular}{|c|c|c|c|}
\hline $\begin{array}{l}\text { Эксперт, } \\
k=1,2\end{array}$ & инцидент на $j=$ & & \\
\hline & 1 & $\mathbf{j}$ & $\mathrm{J}$ \\
\hline 1 & $W_{i\left(6_{1}\right) \Phi^{\text {yгр }}}$ & $W_{i\left(6_{j}\right) \Phi^{\text {угр }}}{ }_{1}$ & 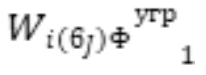 \\
\hline k & $W_{i\left(6_{1}\right) \Phi_{k}}^{\text {yгp }}$ & $W_{i\left(6_{j}\right) \Phi^{\text {yгp }}}{ }_{k}$ & $W_{i\left(6_{j}\right) \Phi^{\text {yгp }}}{ }_{k}$ \\
\hline K & $W_{i\left(6_{1}\right) \Phi^{\text {yгp }}}$ & $W_{i\left(6_{j}\right) \Phi^{\mathrm{y}}{ }_{\mathrm{K}}}^{\mathrm{y} p}$ & $W_{i\left(6_{j}\right) \Phi^{\mathrm{yrp}}}{ }_{\mathrm{K}}$ \\
\hline
\end{tabular}

Рассмотрение методики количественной оценки угрозы с целью обоснования и описания способа определения угрозы на ТКСС и количественной оценки степени этой угрозы рассмотрим при следующих исходных данных:

- временной интервал, на котором оцениваются угрозы реализациинегативных воздействий на TKCC.

- перечень и содержание характерных для этого объекта И.

- перечень и содержание факторов угрозы, которые могут способствовать началу И на ТКСС.

Для конкретных ТКСС содержание исходных данных уточняются специалистами по обеспечению безопасности на основании:
- анализа специфических особенностей этого объекта, характерных для него И, а также факторов, способствующих их реализации;

- накопление опыта борьбы с И;

- имеющейся статистики по происшествиям безопасности применительно к данному объекту и подобным ему объектам безопасности;

- прогнозирования возможных изменений И (например, совершенствование нарушителями способов осуществления актов незаконного вмешательства в работу ТКСС) и факторов, способствующих их реализации.

Ввиду невозможности формализации получения исходных данных количественная оценка угрозы безопасности осуществляется экспертным методом. Выбранной 
группе в составе $K$ экспертов представляется перечень характерных для данной ТКСС И (которые представляют интерес с точки зрения обеспечения безопасности), предлагается определит перечень факторов угрозы воздействия на временном интервале $\Delta \boldsymbol{T}$ и ответить на следующие вопросы:

1. Какие факторы, по вашему мнению, на временном интервале $\Delta \boldsymbol{T}$ могут способствовать угрозе реализации представленных И на данную ТКСС?

2. Какова, по вашему мнению, степень влияния (по шкале $[0,1])$ наличия на интервале $\Delta \boldsymbol{T}$ выбранных вами факторов?

3. Какова, по вашему мнению, степень влияния (по шкале $[0,1])$ каждого из выбранных вами факторов на начало представленных И?

Ответы каждого эксперта фиксируются в форме табл. 1.

По результатам экспертного опроса величины

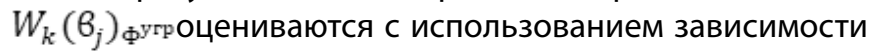
(2) и представляются в форме табл. 2.

В соответствии с данными табл. 2 с использованием зависимостей (5) или (4) проводятся количественные оценки степени угрозы Wi(вj)Tyгpk начала реализации инцидента на $i$-й ОБ (по мнению каждого из участвовавших в опросе экспертов). Результаты этих оценок оформляются в виде табл. 3.

Экспертному методу оценки характеристик СОБ защищаемых объектов принадлежит важная роль в процессе этой оценки вследствие большой сложности структур «ОБ - СОБ», широкого участия в них человека и невозможности исчерпывающего формального описания процессов негативных воздействий на ОБ и противодействия им СОБ.

Экспертный метод, базирующийся на эвристической деятельности человека, является исторически наиболее известным. Эвристическая деятельность представляет собой познавательный процесс, направленный на изучение качественных и количественных сторон исследуемого процесса или явления, протекающий в голове человека с использованием сформированных в мозгу образов этого процесса или явления [9]. Причем зачастую человек не в состоянии четко сформулировать, каким образом он сделал ту или иную оценку.

Совершенно очевидно, что не всякий человек, ежедневно пользующийся своими эвристическими оценками в повседневной жизни, несмотря на прирожденные способности и даже талант, способен количественно оценить характеристики сложных процессов (явлений), когда требуется большой объем знаний в специальных областях и соответствующий опыт работы. Опыт формируется в процессе оценки результатов схожих процессов (явлений), наблюдения за их протеканием, сравнения результатов собственных оценок с практическими результатами и корректировки, исходя из этого, «собственной методики» оценки.

Экспертный метод не потерял своего значения в тех случаях, когда бывает сложно, а иногда на данном уровне знаний просто невозможно построить математическую модель исследуемого процесса, провести оценки формальными методами с требуемой точностью. Между тем человек в подобных ситуациях решает задачи оценки зачастую достаточно успешно и в условиях неполной информации. Здесь проявляется подмеченная Н. Винером замечательная способность человеческого мозга «оперировать с нечетко очерченными понятиями» [10].

В связи с этим, т.к. в основе экспертных оценок, как отмечалось выше, лежит познавательный процесс, протекающий в мозгу человека, они подвержены целому ряду объективных и субъективных факторов, определяемых характером, особенностями мышления и чертами конкретного человека.

Факторами, без которых получение точных оценок просто невозможно, являются уже упомянутые профессиональные знания, опыт, а также интуиция, способность к выделению главного, умение вовремя признать свою ошибку.

К факторам, которые могут отрицательно сказаться на качестве оценки, относятся [10]: профессиональная ограниченность, психологическая инерция, консерватизм мышления, трудность восприятия отрицательных Выводов, склонность к преувеличению плохого, боязнь ответственности.

Влияние на результаты экспертных оценок факторов, обусловленных субъективизмом конкретного человека, может быть умышленно при проведении опроса группы экспертов при правильно организованной процедуре самого опроса и обработке полученных результатов, например, с использованием уже упомянутого метода ДЕЛЬФИ [11].

Метод ДЕЛЬФИ представляет собой многоэтапный экспертный метод, предусматривающий первоначальное изолированное вынесение экспертами своих суждений и дальнейшую многократную их корректировку на базе ознакомления каждого эксперта с суждениями других экспертов до тех пор, пока величина разброса оценок не будет находиться в рамках заранее устанавливаемого желаемого интервала варьирования оцеHOK. 
Таблица 4

\begin{tabular}{|l|l|}
\hline Вид инцидента & Степень угрозы начала инцидента на интервале $\Delta T$ \\
\hline$B_{l}$ & $\bar{W}_{i}\left(B_{1}\right)_{\Phi^{\mathrm{yrp}}}$ \\
\hline$B_{j}$ & $\bar{W}_{i}\left(\theta_{j}\right)_{\Phi^{\mathrm{yrp}}}$ \\
\hline$B_{J}$ & $\bar{W}_{i}\left(6_{J}\right)_{\Phi^{\mathrm{yrp}}}$ \\
\hline
\end{tabular}

$\tilde{y}=\sum_{k=1}^{K} y_{k} / K$

$$
\mathrm{e}=\frac{\left\{\frac{\left[\sum_{k=1}^{K}\left(y_{k}-\tilde{y}\right)^{2}\right]}{K-1}\right\}^{\frac{1}{2}}}{\mathrm{y}}
$$

$$
\bar{W}_{i}\left(6_{i}\right)_{\Phi^{\mathrm{yrp}}}=\sum_{k=1}^{\mathrm{K}} W_{i\left(6_{j}\right) \Phi_{k}^{\mathrm{yrp}}}-K .
$$

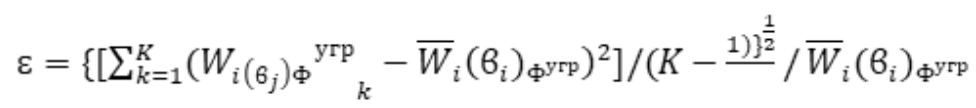

В результате экспертного опроса относительно величины некоторой характеристики СОБ защищаемого объекта обобщенного мнение группы из $K$ экспертов представляется в виде:

- среднего значения величины $y$ (7)

- коэффициента вариации мнений группы экспертов (8)

Результаты опроса подвергаются анализу, целью которого является оценка с помощью коэффициента вариации $\varepsilon$ согласованности мнений экспертов. Если коэффициент вариации не превосходит некоторого заранее оговоренного допустимого значения $\varepsilon^{\text {до }}, \varepsilon \leq \varepsilon^{\text {доn }}$, (9) окончательные результаты принимаются соответствующими оценке (7).

Значение коэффициента $\varepsilon^{\partial o n}$ с учетом случайного характера величины $y$ и возможных ошибок экспертов выбирается в виде некоторого диапазона (для практических задач, например, диапазон 0,15-0,20).
В случае невыполнения условия (9) проводится обсуждение мнений экспертов, находятся причины разброса экспертных оценок и проводится повторный опрос экспертов. Данная процедура проводится до тех пор, пока не будет выполнено условие (9).

С использованием данных табл. 3, находится обобщенное мнение группы экспертов (7) в виде среднего значения степени угрозы начала реализации на интервале $\Delta \boldsymbol{T}$ каждого $j$-го инцидента на $i$-й ОБ (10) и оценивается согласованность мнений группы экспертов (8) по величине коэффициента вариации (11).

В случае наличия согласованного (9) мнения группы экспертов результаты количественной оценки степени угрозы начала реализации на интервале $\Delta \boldsymbol{T}$ инцидента оформляются в виде таблицы 4.

Введение количественного показателя степени угрозы И на ОБ позволяет количественно описать содержа- 
Таблица 5

\begin{tabular}{|c|c|c|c|}
\hline \multirow{2}{*}{$\begin{array}{l}\text { Инцидент, } \boldsymbol{\sigma}_{j} \\
(\boldsymbol{j}=1,2, \ldots, j)\end{array}$} & \multicolumn{3}{|l|}{ Категория угроз $i$-муОБ } \\
\hline & $\begin{array}{l}\text { I категория } \\
\text { угрозы }\end{array}$ & $\begin{array}{l}\text { ІІкатегория } \\
\text { угрозы }\end{array}$ & $\begin{array}{l}\text { III категория } \\
\text { угрозы }\end{array}$ \\
\hline 1 & 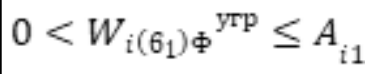 & $A_{i 1}<W_{i\left(6_{1}\right) \Phi}{ }^{\text {yгp }} \leq B_{i 1}$ & $B_{i 1}<W_{i\left(6_{1}\right) \Phi^{\text {yгp }}} \leq 1$ \\
\hline$j$ & 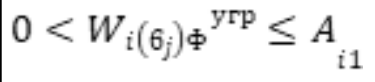 & $A_{i j}<W_{i\left(6_{j}\right) \Phi}^{\text {yгp }} \leq B_{i j}$ & $B_{i j}<W_{i\left(6_{j}\right) \Phi^{\text {yгp }}} \leq 1$ \\
\hline J & $0<W_{i\left(6_{J}\right) \Phi^{\mathrm{y} \Gamma \mathrm{p}} \leq A_{i 1}}$ & 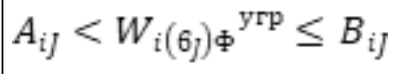 & $B_{i j}<W_{i\left(6_{j}\right) \Phi^{\text {yгp }} \leq 1} \leq 1$ \\
\hline
\end{tabular}

Таблица 6

\begin{tabular}{|c|c|c|c|}
\hline \multirow{2}{*}{ HB } & \multicolumn{3}{|l|}{ Категория угроз $\boldsymbol{i}$-муОБ } \\
\hline & Потенциальная & Непосредственная & Прямая \\
\hline$B_{j}$ & $0<W_{i\left(6_{J}\right) \Phi^{\text {yгp }} \leq 0,30}$ & $0,30<W_{i\left(6_{j}\right) \Phi^{y г p}} \leq 0,80$ & $0,80<W_{i\left(6_{j}\right) \Phi}{ }^{\text {угр }} \leq 1$ \\
\hline
\end{tabular}

ние различных существующих качественных категорий угроз («красной», «оранжевой», «желтой», «прямой», «непосредственной» и т.п.).

Указанные категории угроз могут быть заданы табл. 5, в которой интервалы значений $(A i j, B i j)$ вероятности $W i(в j) T y г p$ для каждого $i$-го ОБ применительно к $j$-му инциденту задаются экспертами.

В частности, границы диапазона значений вероятностей Wi(вj)Tyгр могут быть такими:

$$
A_{i j}=0,20-0,30 ; B_{i j}=0,80-0,85 \text { (12) }
$$

Так введение показателя степени угрозы И на ОТИ и ТС позволяет, количественно определить понятие степени угрозы совершения незаконного вмешательства в деятельность объектов ТКСС.
Принимая во внимание уровни безопасности объектов ТКСС и порядок их объявления (установления) получим качественную классификацию угроз (I- потенциальная угроза, II-непосредственная угроза, III - прямая угроза) и количественные значения диапазона вероятностей (12), табл. 5 для И- $a$ в рассматриваемом случае может представляться в виде табл. 6.

В этом случае оцененную угрозу начала реализации И- $a$, характеризуемую вероятностью $\bar{W}_{i}\left(b_{1}\right)_{\Phi \text { угр }}=0,86$, можно отнести к категории «прямая угроза».

Предложенная методика количественной оценки угрозы начала реализации И на ОБ, может быть использована для принятия научно-обоснованных решений по их защите и повышении эффективности системы управления в информационно-телекоммуникационных системах [12].

\section{ЛИТЕРАТУРА}

1. Федеральный закон от 5 марта1992 г. № 2446-1 «0 безопасности».

2. ГОСТ Р ИСО/МЭК 13335-1-206 // Методы и средства обеспечения безопасности. Концепция и модели менеджмента безопасности информационных и телекоммуникационных технологий.

3. Пелешенко В.С., Говорова С. В., Лапина М.А. //Менеджмент инцидентов информационной безопасности защищенных автоматизированных систем управления: Ставрополь: СКФУ, 2017.

4. Михайлов Ю.Б. // Математические основы повышения точности прогнозирования количественных характеристик процессов. М.: Научтехлитиздат, 2000;

5. Смирнов Н. В., Дунин-Барковский И.В. // Курс теории вероятностей и математической статистики. М.: Наука, 1965;

6. Бусленко Н. П. и др. // Метод статистических испытаний. М.: Сов. Радио, 1972. 
7. Вентцель Е. С. Исследование операций. М.: Сов. Радио, 1972.

8. Семенов В. А. // Теория вероятностей и математическая статистика: Учебное пособие. Стандарт третьего поколения. СПб.: Питер, 2013.

9. Орлов А. И. // Экспертные оценки. Учебное пособие. М., 2002.

10. Чуев Ю. В., Михайлов Ю. Б., Кузьмин В. И. // Прогнозирование количественных характеристик процессов. М.: Сов. Радио, 1971.

11. А. Г. Гранберг // Статистическое моделирование и прогнозирование. М.: Финансы и статистика, 1990.

12. Шилина А. Н., Кузнецова В.В., Гайдаревский А. А. // Предложения по оптимизации показателей эффективности системы управления в информационно-телекоммуникационных систем // Материалы III международной научно-практической конференции «Фундаментально-прикладные проблемы безопасности, живучести, надежности, устойчивости и эффективности систем» Часть I, Елец-2019.

( ) Назарова Ольга Юрьевна ( olga2018rostov@yandex.ru ), Сагдеев Александр Константинович ( brother-aks@yandex.ru ),

Стахеев Иван Геннадиевич, Титова Ольга Викторовна, Шилина Анна Николаевна.

Журнал «Современная наука: актуальные проблемы теории и практики»

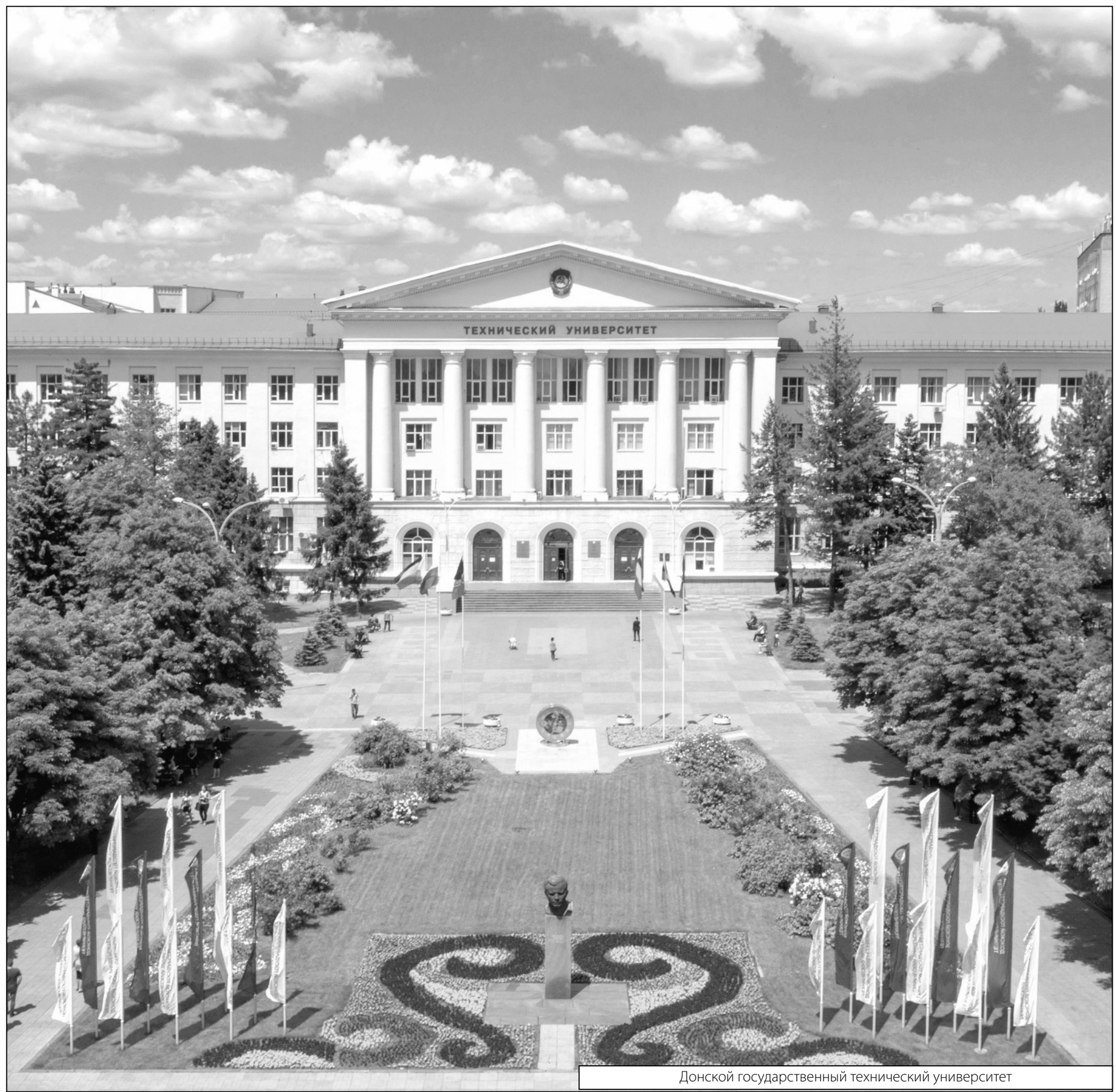

